\title{
Projection algorithms for treating asymptotically quasi- $\phi$-nonexpansive mappings in the intermediate sense
}

Yuan Hecai ${ }^{*}$ and Liu Aichao ${ }^{2}$

\section{"Correspondence:}

hsyuanhc@yeah.net

'School of Mathematics and

Information Science, North China

University of Water Resources and

Electric Power, Zhengzhou, 450011

China

Full list of author information is

available at the end of the article

\begin{abstract}
In this paper, we investigate the fixed point problem of asymptotically quasi- $\phi$-nonexpansive mappings in the intermediate sense based on a projection algorithm. Strong convergence of the proposed algorithm is obtained in a reflexive, strictly convex, and smooth Banach space.
\end{abstract}

MSC: $47 \mathrm{H} 09 ; 47 J 25$

Keywords: asymptotically quasi- $\phi$-nonexpansive mapping in the intermediate sense; relatively nonexpansive mapping; generalized projection; fixed point

\section{Introduction}

Fixed point theory of nonlinear mapping is a popular research topic of common interest in two areas of nonlinear analysis and optimization. Over the last 60 years or so, the theory of fixed points has been revealed as a very powerful and important tool in the study of nonlinear phenomena. In particular, fixed point techniques have been applied in such diverse fields as biology, chemistry, economics, engineering, game theory, and physics; see [1-11] and the references therein. There are many results on the existence of fixed points of nonlinear mappings. However, from the standpoint of real world applications, it is not only to know the existence of fixed points of nonlinear mappings, but also to be able to construct an iterative algorithm to approximate their fixed points. The computation of fixed points is important in the study of many real world problems, including inverse problems; for instance, it is not hard to show that the split feasibility problem and the convex feasibility problem in signal processing and image reconstruction can both be formulated as a problem of finding fixed points of certain operators, respectively; see $[1,2]$ for more details and the references therein.

For iterative algorithms, the oldest and simplest one is the Picard iterative algorithm. It is known that $T$, where $T$ stands for a contractive mapping, enjoys a unique fixed point, and the sequence generated by the Picard iterative algorithm can converge to the unique fixed point. However, for more general nonexpansive mappings, the Picard iterative algorithm fails to converge to fixed points of nonexpansive mappings even if they enjoy fixed points. The Krasnoselskii-Mann iterative algorithm has been studied for approximating fixed points of nonexpansive mappings and their extensions. However, the KrasnoselskiiMann iterative algorithm is weak convergence for nonexpansive mappings only; see [12]. In many disciplines, problems arise in infinite dimension spaces. In such problems, strong 
convergence (norm convergence) is often much more desirable than weak convergence for it translates the physically tangible property so that the energy $\left\|x_{n}-x\right\|$ of the error between the iterate $x_{n}$ and the solution $x$ eventually becomes arbitrarily small. Strong convergence of iterative sequences properties has a direct impact when the process is executed directly in the underlying infinite dimensional space.

Projection methods which were first introduced by Haugazeau [13] have been considered for the approximation of fixed points of nonlinear mappings. The advantage of projection methods is that strong convergence of iterative sequences can be guaranteed without any compact assumptions.

The purpose of this paper is to investigate a projection algorithm for asymptotically quasi- $\phi$-nonexpansive mappings in the intermediate sense. The organization of this paper is as follows. In Section 2, we provide some necessary preliminaries. In Section 3, a projection algorithm is investigated. Strong convergence of the purposed algorithm is obtained in a reflexive, strictly convex, and smooth Banach space. Some deduced results are also obtained.

\section{Preliminaries}

Let $E$ be a real Banach space, $C$ be a nonempty subset of $E$ and $T: C \rightarrow C$ be a nonlinear mapping. In this paper, we use $F(T)$ to denote the fixed point set of $T$. The mapping $T$ is said to be asymptotically regular on $C$ if for any bounded subset $K$ of $C$,

$$
\limsup _{n \rightarrow \infty}\left\{\left\|T^{n+1} x-T^{n} x\right\|: x \in K\right\}=0 .
$$

The mapping $T$ is said to be closed if for any sequence $\left\{x_{n}\right\} \subset C$ such that $\lim _{n \rightarrow \infty} x_{n}=x_{0}$ and $\lim _{n \rightarrow \infty} T x_{n}=y_{0}$, then $T x_{0}=y_{0}$. A point $x \in C$ is a fixed point of $T$ provided $T x=x$. In this paper, we use $\rightarrow$ and $\rightarrow$ to denote the strong convergence and weak convergence, respectively.

Recall that the mapping $T$ is said to be nonexpansive iff

$$
\|T x-T y\| \leq\|x-y\|, \quad \forall x, y \in C .
$$

$T$ is said to be quasi-nonexpansive iff $F(T) \neq \emptyset$, and

$$
\|p-T y\| \leq\|p-y\|, \quad \forall p \in F(T), \forall y \in C .
$$

$T$ is said to be asymptotically nonexpansive iff there exists a sequence $\left\{k_{n}\right\} \subset[1, \infty)$ with $k_{n} \rightarrow 1$ as $n \rightarrow \infty$ such that

$$
\left\|T^{n} x-T^{n} y\right\| \leq k_{n}\|x-y\|, \quad \forall x, y \in C, \forall n \geq 1
$$

$T$ is said to be asymptotically quasi-nonexpansive iff $F(T) \neq \emptyset$ and there exists a sequence $\left\{k_{n}\right\} \subset[1, \infty)$ with $k_{n} \rightarrow 1$ as $n \rightarrow \infty$ such that

$$
\left\|p-T^{n} y\right\| \leq k_{n}\|p-y\|, \quad \forall p \in F(T), \forall y \in C, \forall n \geq 1 .
$$

The class of asymptotically nonexpansive mappings was introduced by Goebel and Kirk [14] in 1972. In uniformly convex Banach spaces, they proved that if $C$ is nonempty bounded closed and convex, then every asymptotically nonexpansive self-mapping $T$ on 
$C$ has a fixed point. Further, the fixed point set of $T$ is closed and convex. Since 1972, a host of authors have studied the weak and strong convergence of iterative algorithms for such a class of mappings.

$T$ is said to be asymptotically nonexpansive in the intermediate sense iff it is continuous and the following inequality holds:

$$
\limsup _{n \rightarrow \infty} \sup _{x, y \in C}\left(\left\|T^{n} x-T^{n} y\right\|-\|x-y\|\right) \leq 0 .
$$

$T$ is said to be asymptotically quasi-nonexpansive in the intermediate sense iff $F(T) \neq \emptyset$ and the following inequality holds:

$$
\limsup _{n \rightarrow \infty} \sup _{p \in F(T), y \in C}\left(\left\|p-T^{n} y\right\|-\|p-y\|\right) \leq 0 .
$$

The class of the mappings which are asymptotically nonexpansive in the intermediate sense was considered by Bruck et al. [15]. It is worth mentioning that the class of mappings which are asymptotically nonexpansive in the intermediate sense may not be Lipschitz continuous. However, asymptotically nonexpansive mappings are Lipschitz continuous.

One of classical iterations is the Halpern iteration [16] which generates a sequence in the following manner:

$$
\forall x_{1} \in C, \quad x_{n+1}=\alpha_{n} u+\left(1-\alpha_{n}\right) T x_{n}, \quad \forall n \geq 1,
$$

where $\left\{\alpha_{n}\right\}$ is a sequence in the interval $(0,1)$ and $u \in C$ is a fixed element.

Since 1967 , the Halpern iteration has been studied extensively by many authors. It is well known that the following two restrictions:

(C1) $\lim _{n \rightarrow \infty} \alpha_{n}=0$;

(C2) $\sum_{n=1}^{\infty} \alpha_{n}=\infty$

are necessary in the sense that if the Halbern iterative sequence is strongly convergent for all nonexpansive self-mappings defined on $C$. To improve the rate of convergence of the Halbern iterative sequence, we cannot rely only on the iteration itself. Hybrid projection methods recently have been applied to solve the problem.

Martinez-Yanes and $\mathrm{Xu}$ [17] considered the hybrid projection algorithm for a nonexpansive mapping in a Hilbert space. Strong convergence theorems are established under the condition $(\mathrm{C} 1)$ only imposed on the control sequence. To be more precise, they proved the following theorem.

Theorem 2.1 Let $H$ be a real Hilbert space, $C$ be a closed convex subset of $H$ and $T: C \rightarrow$ $C$ be a nonexpansive mapping such that $F(T) \neq \emptyset$. Assume that $\left\{\alpha_{n}\right\} \subset(0,1)$ is such that $\lim _{n \rightarrow \infty} \alpha_{n}=0$. Then the sequence $\left\{x_{n}\right\}$ defined by

$$
\left\{\begin{array}{l}
x_{0} \in C \quad \text { chosen arbitrarily, } \\
y_{n}=\alpha_{n} x_{0}+\left(1-\alpha_{n}\right) T x_{n}, \\
C_{n}=\left\{z \in C:\left\|y_{n}-z\right\|^{2} \leq\left\|x_{n}-z\right\|^{2}+\alpha_{n}\left(\left\|x_{0}\right\|^{2}+2\left\langle x_{n}-x_{0}, z\right\rangle\right)\right\} \\
Q_{n}=\left\{z \in C:\left\langle x_{0}-x_{n}, x_{n}-z\right\rangle \geq 0\right\} \\
x_{n+1}=P_{C_{n} \cap Q_{n}} x_{0}, \quad \forall n \geq 0
\end{array}\right.
$$

converges strongly to $P_{F(T)} x_{0}$. 
Let $E$ be a Banach space with the dual $E^{*}$. We denote by $J$ the normalized duality mapping from $E$ to $2^{E^{*}}$ defined by

$$
J x=\left\{f^{*} \in E^{*}:\left\langle x, f^{*}\right\rangle=\|x\|^{2}=\left\|f^{*}\right\|^{2}\right\},
$$

where $\langle\cdot, \cdot\rangle$ denotes the generalized duality pairing.

A Banach space $E$ is said to be strictly convex if $\left\|\frac{x+y}{2}\right\|<1$ for all $x, y \in E$ with $\|x\|=$ $\|y\|=1$ and $x \neq y$. It is said to be uniformly convex if $\lim _{n \rightarrow \infty}\left\|x_{n}-y_{n}\right\|=0$ for any two sequences $\left\{x_{n}\right\}$ and $\left\{y_{n}\right\}$ in $E$ such that $\left\|x_{n}\right\|=\left\|y_{n}\right\|=1$ and $\lim _{n \rightarrow \infty}\left\|\frac{x_{n}+y_{n}}{2}\right\|=1$. Let $U_{E}=\{x \in E:\|x\|=1\}$ be the unit sphere of $E$. Then the Banach space $E$ is said to be smooth provided

$$
\lim _{t \rightarrow 0} \frac{\|x+t y\|-\|x\|}{t}
$$

exists for each $x, y \in U_{E}$. It is also said to be uniformly smooth if the above limit is attained uniformly for $x, y \in U_{E}$. It is well known that if $E$ is uniformly smooth, then $J$ is uniformly norm-to-norm continuous on each bounded subset of $E$. It is also well known that $E$ is uniformly smooth if and only if $E^{*}$ is uniformly convex.

Recall that a Banach space $E$ enjoys the Kadec-Klee property if for any sequence $\left\{x_{n}\right\} \subset E$, and $x \in E$ with $x_{n} \rightarrow x$, and $\left\|x_{n}\right\| \rightarrow\|x\|$, then $\left\|x_{n}-x\right\| \rightarrow 0$ as $n \rightarrow \infty$. For more details on the Kadec-Klee property, the readers can refer to [18] and the references therein. It is well known that if $E$ is a uniformly convex Banach space, then $E$ enjoys the Kadec-Klee property.

As we all know, if $C$ is a nonempty closed convex subset of a Hilbert space $H$ and $P_{C}: H \rightarrow C$ is the metric projection of $H$ onto $C$, then $P_{C}$ is nonexpansive. This fact actually characterizes Hilbert spaces, and consequently, it is not available in more general Banach spaces. In this connection, Alber [19] recently introduced a generalized projection operator $\Pi_{C}$ in a Banach space $E$ which is an analogue of the metric projection in Hilbert spaces.

Next, we assume that $E$ is a smooth Banach space. Consider the functional defined by

$$
\phi(x, y)=\|x\|^{2}-2\langle x, J y\rangle+\|y\|^{2} \quad \text { for } x, y \in E .
$$

Observe that, in a Hilbert space $H$, (2.1) is reduced to $\phi(x, y)=\|x-y\|^{2}, x, y \in H$. The generalized projection $\Pi_{C}: E \rightarrow C$ is a map that assigns to an arbitrary point $x \in E$ the minimum point of the functional $\phi(x, y)$, that is, $\Pi_{C} x=\bar{x}$, where $\bar{x}$ is the solution to the minimization problem

$$
\phi(\bar{x}, x)=\min _{y \in C} \phi(y, x)
$$

the existence and uniqueness of the operator $\Pi_{C}$ follow from the properties of the functional $\phi(x, y)$ and strict monotonicity of the mapping $J$; for more details, see [18] and [19] and the references therein. In Hilbert spaces, $\Pi_{C}=P_{C}$. It is obvious from the definition of a function $\phi$ that

$$
(\|x\|-\|y\|)^{2} \leq \phi(x, y) \leq(\|y\|+\|x\|)^{2}, \quad \forall x, y \in E,
$$


and

$$
\phi(x, y)=\phi(x, z)+\phi(z, y)+2\langle x-z, J z-J y\rangle, \quad \forall x, y, z \in E
$$

Remark 2.2 If $E$ is a reflexive, strictly convex, and smooth Banach space, then for $x, y \in E$, $\phi(x, y)=0$ if and only if $x=y$. It is sufficient to show that if $\phi(x, y)=0$, then $x=y$. From (2.2), we have $\|x\|=\|y\|$. This implies that $\langle x, J y\rangle=\|x\|^{2}=\|J y\|^{2}$. From the definition of $J$, we have $J x=J y$. Therefore, we have $x=y$; for more details, see [18] and [19] and the references therein.

Let $C$ be a nonempty closed convex subset of $E$ and let $T$ be a mapping from $C$ into itself. A point $p$ in $C$ is said to be an asymptotic fixed point of $T$ [20] if $C$ contains a sequence $\left\{x_{n}\right\}$ which converges weakly to $p$ such that $\lim _{n \rightarrow \infty}\left\|x_{n}-T x_{n}\right\|=0$. The set of asymptotic fixed points of $T$ will be denoted by $\widetilde{F}(T)$. A mapping $T$ from $C$ into itself is said to be relatively nonexpansive [21] if $\widetilde{F}(T)=F(T) \neq \emptyset$ and $\phi(p, T x) \leq \phi(p, x)$ for all $x \in C$ and $p \in F(T)$. The mapping $T$ is said to be relatively asymptotically nonexpansive $[22,23]$ if $\widetilde{F}(T)=F(T) \neq \emptyset$ and there exists a sequence $\left\{k_{n}\right\} \subset[1, \infty)$ with $k_{n} \rightarrow 1$ as $n \rightarrow \infty$ such that $\phi(p, T x) \leq k_{n} \phi(p, x)$ for all $x \in C, p \in F(T)$ and $n \geq 1$.

The mapping $T$ is said to be quasi- $\phi$-nonexpansive [24] if $F(T) \neq \emptyset$ and $\phi(p, T x) \leq \phi(p, x)$ for all $x \in C$ and $p \in F(T)$. $T$ is said to be asymptotically quasi- $\phi$-nonexpansive [25-27] if $F(T) \neq \emptyset$ and there exists a sequence $\left\{k_{n}\right\} \subset[0, \infty)$ with $k_{n} \rightarrow 1$ as $n \rightarrow \infty$ such that $\phi(p, T x) \leq k_{n} \phi(p, x)$ for all $x \in C, p \in F(T)$ and $n \geq 1$.

Remark 2.3 The class of asymptotically quasi- $\phi$-nonexpansive mappings is more general than the class of relatively asymptotically nonexpansive mappings which requires the restriction $F(T)=\widetilde{F}(T)$.

In 2007, Qin and Su [28] extended the results of Martinez-Yanes and Xu [17] from Hilbert spaces to Banach spaces. To be more precise, they established the following theorem.

Theorem 2.4 Let $E$ be a uniformly convex and uniformly smooth Banach space, let $C$ be a nonempty closed convex subset of $E$ and let $T: C \rightarrow C$ be a relatively nonexpansive mapping. Assume that $\left\{\alpha_{n}\right\}$ is a sequence in $(0,1)$ such that $\lim _{n \rightarrow \infty} \alpha_{n}=0$. Define a sequence $\left\{x_{n}\right\}$ in $C$ in the following manner:

$$
\left\{\begin{array}{l}
x_{0} \in C \quad \text { chosen arbitrarily, } \\
y_{n}=J^{-1}\left(\alpha_{n} J x_{0}+\left(1-\alpha_{n}\right) J T x_{n}\right), \\
C_{n}=\left\{v \in C: \phi\left(v, y_{n}\right) \leq \alpha_{n} \phi\left(v, x_{0}\right)+\left(1-\alpha_{n}\right) \phi\left(v, x_{n}\right)\right\}, \\
Q_{n}=\left\{v \in C:\left\langle J x_{0}-J x_{n}, x_{n}-v\right\rangle \geq 0\right\}, \\
x_{n+1}=\Pi_{C_{n} \cap Q_{n}} x_{0}, \quad \forall n \geq 0 .
\end{array}\right.
$$


$T$ is said to be an asymptotically quasi- $\phi$-nonexpansive in the intermediate sense [29] if $F(T) \neq \emptyset$ and

$$
\limsup _{n \rightarrow \infty} \sup _{p \in F(T), x \in C}\left(\phi\left(p, T^{n} x\right)-\phi(p, x)\right) \leq 0
$$

Put

$$
\xi_{n}=\max \left\{0, \sup _{p \in F(T), x \in C}\left(\phi\left(p, T^{n} x\right)-\phi(p, x)\right)\right\} .
$$

It follows that $\xi_{n} \rightarrow 0$ as $n \rightarrow \infty$. Then (2.4) is reduced to the following:

$$
\phi\left(p, T^{n} x\right) \leq \phi(p, x)+\xi_{n}, \quad \forall p \in F(T), \forall x \in C .
$$

Remark 2.5 The class of asymptotically quasi- $\phi$-nonexpansive mappings in the intermediate sense is a generalization of the class of asymptotically quasi-nonexpansive mappings in the intermediate sense in the framework of Banach spaces.

Recently, many authors investigated fixed point problems of asymptotically quasi- $\phi$ nonexpansive mappings and asymptotically quasi- $\phi$-nonexpansive mappings in the intermediate sense based on projection algorithms; for more details, see [30-36]. In this paper, we investigate the fixed point problems of asymptotically quasi- $\phi$-nonexpansive mappings in the intermediate sense based on a projection algorithm. Strong convergence of the proposed algorithm is obtained in a reflexive, strictly convex, and smooth Banach space such that both $E$ and $E^{*}$ have the Kadec-Klee property. The results presented in this paper mainly improve the corresponding results in Cho et al. [30].

In order to prove our main results, we need the following lemmas.

Lemma 2.6 [18] Let C be a nonempty, closed, and convex subset of a smooth Banach space $E$, and $x \in E$. Then $x_{0}=\Pi_{C} x$ if and only if

$$
\left\langle x_{0}-y, J x-J x_{0}\right\rangle \geq 0, \quad \forall y \in C
$$

Lemma 2.7 [18] Let E be a reflexive, strictly, convex, and smooth Banach space, let $C$ be a nonempty, closed, and convex subset of $E$, and $x \in E$. Then

$$
\phi\left(y, \Pi_{C} x\right)+\phi\left(\Pi_{C} x, x\right) \leq \phi(y, x), \quad \forall y \in C .
$$

\section{Main results}

Theorem 3.1 Let E be a reflexive, strictly convex, and smooth Banach space such that both $E$ and $E^{*}$ have the Kadec-Klee property. Let $C$ be a nonempty closed and convex subset of $E$. Let $T: C \rightarrow C$ be a closed and asymptotically quasi- $\phi$-nonexpansive mapping in the intermediate sense. Assume that $T$ is asymptotically regular on $C$ and $F(T)$ is nonempty 
and bounded. Let $\left\{x_{n}\right\}$ be a sequence generated in the following manner:

$$
\left\{\begin{array}{l}
x_{0} \in E \quad \text { chosen arbitrarily, } \\
C_{1}=C, \\
x_{1}=\Pi_{C_{1} x_{0}}, \\
y_{n}=J^{-1}\left(\alpha_{n} J x_{1}+\left(1-\alpha_{n}\right) J T^{n} x_{n}\right), \\
C_{n+1}=\left\{z \in C_{n}: \phi\left(z, y_{n}\right) \leq \phi\left(z, x_{n}\right)+\alpha_{n}\left(\left\|x_{1}\right\|^{2}+2\left\langle z, J x_{n}-J x_{1}\right\rangle\right)+\xi_{n}\right\}, \\
x_{n+1}=\Pi_{C_{n+1}} x_{1},
\end{array}\right.
$$

where

$$
\xi_{n}=\max \left\{0, \sup _{p \in F(T), x \in C}\left(\phi\left(p, T^{n} x\right)-\phi(p, x)\right)\right\} .
$$

If the sequence $\left\{\alpha_{n}\right\}$ satisfies the restriction $\lim _{n \rightarrow \infty} \alpha_{n}=0$, then the sequence $\left\{x_{n}\right\}$ converges strongly to $\Pi_{F(T)} x_{1}$, where $\Pi_{F(T)}$ is the generalized projection from $C$ onto $F(T)$.

Proof First, we show that $F(T)$ is closed and convex. Since $T$ is closed, we can easily conclude that $F(T)$ is also closed. This proves that $F(T)$ is closed. Next, we prove the convexness of $F(T)$. Let $p_{1}, p_{2} \in F(T)$, and $p=t p_{1}+(1-t) p_{2}$, where $t \in(0,1)$. We see that $p=T p$. Indeed, we see from the definition of $T$ that

$$
\phi\left(p_{1}, T^{n} p\right) \leq \phi\left(p_{1}, p\right)+\xi_{n}
$$

and

$$
\phi\left(p_{2}, T^{n} p\right) \leq \phi\left(p_{2}, p\right)+\xi_{n} .
$$

In view of (2.2), we find that

$$
\phi\left(p_{1}, T^{n} p\right)=\phi\left(p_{1}, p\right)+\phi\left(p, T^{n} p\right)+2\left\langle p_{1}-p, J p-J T^{n} p\right\rangle
$$

and

$$
\phi\left(p_{2}, T^{n} p\right)=\phi\left(p_{2}, p\right)+\phi\left(p, T^{n} p\right)+2\left\langle p_{2}-p, J p-J T^{n} p\right\rangle .
$$

Combining (3.1), (3.2), (3.3) with (3.4) yields that

$$
\phi\left(p, T^{n} p\right) \leq 2\left\langle p-p_{1}, J p-J T^{n} p\right\rangle+\xi_{n}
$$

and

$$
\phi\left(p, T^{n} p\right) \leq 2\left\langle p-p_{2}, J p-J T^{n} p\right\rangle+\xi_{n} .
$$

Multiplying $t$ and $(1-t)$ on the both sides of (3.5) and (3.6), respectively, yields that

$$
\lim _{n \rightarrow \infty} \phi\left(p, T^{n} p\right)=0
$$


In light of (2.3), we arrive at

$$
\lim _{n \rightarrow \infty}\left\|T^{n} p\right\|=\|p\|
$$

It follows that

$$
\lim _{n \rightarrow \infty}\left\|J\left(T^{n} p\right)\right\|=\|J p\|
$$

Since $E^{*}$ is reflexive, we may, without loss of generality, assume that $J\left(T^{n} p\right) \rightarrow e^{*} \in E^{*}$. In view of the reflexivity of $E$, we have $J(E)=E^{*}$. This shows that there exists an element $e \in E$ such that $J e=e^{*}$. It follows that

$$
\phi\left(p, T^{n} p\right)=\|p\|^{2}-2\left\langle p, J\left(T^{n} p\right)\right\rangle+\left\|T^{n} p\right\|^{2}=\|p\|^{2}-2\left\langle p, J\left(T^{n} p\right)\right\rangle+\left\|J\left(T^{n} p\right)\right\|^{2} .
$$

Taking $\liminf \operatorname{in}_{n \rightarrow \infty}$ on the both sides of the equality above, we obtain that

$$
\begin{aligned}
0 & \geq\|p\|^{2}-2\left\langle p, e^{*}\right\rangle+\left\|e^{*}\right\|^{2} \\
& =\|p\|^{2}-2\langle p, J e\rangle+\|J e\|^{2} \\
& =\|p\|^{2}-2\langle p, J e\rangle+\|e\|^{2} \\
& =\phi(p, e) .
\end{aligned}
$$

This implies that $p=e$, that is, $J p=e^{*}$. It follows that $J\left(T^{n} p\right) \rightarrow J p \in E^{*}$. In view of the Kadec-Klee property of $E^{*}$, we obtain from (3.8) that $\lim _{n \rightarrow \infty}\left\|J\left(T^{n} p\right)-J p\right\|=0$. Since $J^{-1}$ : $E^{*} \rightarrow E$ is demicontinuous, we see that $T^{n} p \rightarrow p$. By virtue of the Kadec-Klee property of $E$, we see from (3.7) that $T^{n} p \rightarrow p$ as $n \rightarrow \infty$. Hence $T T^{n} p=T^{n+1} p \rightarrow p$ as $n \rightarrow \infty$. In view of the closedness of $T$, we can obtain that $p \in F(T)$. This shows that $F(T)$ is convex. This completes the proof that $F(T)$ is convex and closed. This means that $\Pi_{F(T)} x$ is well defined for any $x \in C$. Next, we show that $C_{n}$ is closed and convex. It is obvious that $C_{1}=C$ is closed and convex. Suppose that $C_{h}$ is closed and convex for some $h \in \mathbb{N}$. For $z \in C_{h}$, we see that

$$
\phi\left(z, y_{h}\right) \leq \phi\left(z, x_{h}\right)+\alpha_{h}\left(\left\|x_{1}\right\|^{2}+2\left\langle z, J x_{h}-J x_{1}\right\rangle\right)+\xi_{h}
$$

is equivalent to

$$
2\left\langle z, J x_{h}-J y_{h}\right\rangle+2 \alpha_{h}\left\langle z, J x_{1}-J x_{h}\right\rangle \leq\left\|x_{h}\right\|^{2}-\left\|y_{h}\right\|^{2}+\alpha_{h}\left\|x_{1}\right\|^{2}+\xi_{h} .
$$

It is not hard to see that $C_{h+1}$ is closed and convex. Then, for each $n \geq 1, C_{n}$ is closed and convex. This shows that $\Pi_{C_{n+1}} x_{1}$ is well defined.

Next, we prove that $\mathcal{F} \subset C_{n} . F(T) \subset C_{1}=C$ is obvious. Suppose that $F(T) \subset C_{h}$ for some $h \in \mathbb{N}$. Then, for $\forall w \in F(T) \subset C_{h}$, we have

$$
\begin{aligned}
\phi\left(w, y_{h}\right) & =\phi\left(w, J^{-1}\left(\alpha_{h} J x_{1}+\left(1-\alpha_{h}\right) J T^{h} x_{h}\right)\right) \\
& =\|w\|^{2}-2\left\langle w, \alpha_{h} J x_{1}+\left(1-\alpha_{h}\right) J T^{h} x_{h}\right\rangle+\left\|\alpha_{h} J x_{1}+\left(1-\alpha_{h}\right) J T^{h} x_{h}\right\|^{2}
\end{aligned}
$$




$$
\begin{aligned}
& \leq\|w\|^{2}-2 \alpha_{h}\left\langle w, J x_{1}\right\rangle-2\left(1-\alpha_{h}\right)\left\langle w, J T^{h} x_{h}\right\rangle+\alpha_{h}\left\|x_{1}\right\|^{2}+\left(1-\alpha_{h}\right)\left\|T^{h} x_{h}\right\|^{2} \\
& =\alpha_{h} \phi\left(w, x_{1}\right)+\left(1-\alpha_{h}\right) \phi\left(w, T^{h} x_{h}\right) \\
& \leq \phi\left(w, x_{h}\right)+\alpha_{h}\left(\phi\left(w, x_{1}\right)-\phi\left(w, x_{h}\right)\right)+\left(1-\alpha_{h}\right) \xi_{n} \\
& \leq \phi\left(w, x_{h}\right)+\alpha_{h}\left(\left\|x_{1}\right\|^{2}+2\left\langle z, J x_{h}-J x_{1}\right\rangle\right)+\xi_{n} .
\end{aligned}
$$

This shows that $w \in C_{h+1}$. This implies that $\mathcal{F} \subset C_{n}$. In view of $x_{n}=\Pi_{C_{n}} x_{1}$, we see that

$$
\left\langle x_{n}-z, J x_{1}-J x_{n}\right\rangle \geq 0, \quad \forall z \in C_{n} .
$$

Since $F(T) \subset C_{n}$, we arrive at

$$
\left\langle x_{n}-w, J x_{1}-J x_{n}\right\rangle \geq 0, \quad \forall w \in F(T) .
$$

It follows from Lemma 2.7 that

$$
\begin{aligned}
\phi\left(x_{n}, x_{1}\right) & =\phi\left(\Pi_{C_{n}} x_{1}, x_{1}\right) \\
& \leq \phi\left(\Pi_{F(T)} x_{1}, x_{1}\right)-\phi\left(\Pi_{F(T)} x_{1}, x_{n}\right) \\
& \leq \phi\left(\Pi_{F(T)} x_{1}, x_{1}\right) .
\end{aligned}
$$

This implies that the sequence $\left\{\phi\left(x_{n}, x_{0}\right)\right\}$ is bounded. It follows from (2.2) that the sequence $\left\{x_{n}\right\}$ is also bounded. Now, we are in a position to show that $x_{n} \rightarrow \bar{x}$, where $\bar{x} \in F(T)$ as $n \rightarrow \infty$. Since $\left\{x_{n}\right\}$ is bounded, and the space is reflexive, we may assume that $x_{n} \rightarrow \bar{x}$. Since $C_{n}$ is closed and convex, we see that $\bar{x} \in C_{n}$. On the other hand, we see from the weakly lower semicontinuity of the norm that

$$
\begin{aligned}
\phi\left(\bar{x}, x_{1}\right) & =\|\bar{x}\|^{2}-2\left\langle\bar{x}, J x_{1}\right\rangle+\left\|x_{1}\right\|^{2} \\
& \leq \liminf _{n \rightarrow \infty}\left(\left\|x_{n}\right\|^{2}-2\left\langle x_{n}, J x_{1}\right\rangle+\left\|x_{1}\right\|^{2}\right) \\
& =\liminf _{n \rightarrow \infty} \phi\left(x_{n}, x_{1}\right) \\
& \leq \limsup _{n \rightarrow \infty} \phi\left(x_{n}, x_{1}\right) \\
& \leq \phi\left(\bar{x}, x_{1}\right),
\end{aligned}
$$

which implies that $\lim _{n \rightarrow \infty} \phi\left(x_{n}, x_{1}\right)=\phi\left(\bar{x}, x_{1}\right)$. Hence, we have $\lim _{n \rightarrow \infty}\left\|x_{n}\right\|=\|\bar{x}\|$. In view of the Kadec-Klee property of $E$, we see that $x_{n} \rightarrow \bar{x}$ as $n \rightarrow \infty$. Next, we show that $\bar{x} \in F(T)$. Since $x_{n}=\Pi_{C_{n}} x_{1}$ and $x_{n+1}=\Pi_{C_{n+1}} x_{1} \in C_{n+1} \subset C_{n}$, we find that $\phi\left(x_{n}, x_{1}\right) \leq$ $\phi\left(x_{n+1}, x_{1}\right)$. This shows that $\left\{\phi\left(x_{n}, x_{1}\right)\right\}$ is nondecreasing. It follows from the boundedness that $\lim _{n \rightarrow \infty} \phi\left(x, x_{1}\right)$ exists. In view of construction of $x_{n+1}=\Pi_{C_{n+1}} x_{1} \in C_{n+1} \subset C_{n}$, we arrive at

$$
\begin{aligned}
\phi\left(x_{n+1}, x_{n}\right) & =\phi\left(x_{n+1}, \Pi_{C_{n}} x_{1}\right) \\
& \leq \phi\left(x_{n+1}, x_{1}\right)-\phi\left(\Pi_{C_{n}} x_{1}, x_{1}\right) \\
& =\phi\left(x_{n+1}, x_{1}\right)-\phi\left(x_{n}, x_{1}\right) .
\end{aligned}
$$


It follows that

$$
\lim _{n \rightarrow \infty} \phi\left(x_{n+1}, x_{n}\right)=0
$$

Since $x_{n+1}=\Pi_{C_{n+1}} x_{1} \in C_{n+1}$, we arrive at

$$
\phi\left(x_{n+1}, y_{n}\right) \leq \phi\left(x_{n+1}, x_{n}\right)+\alpha_{n}\left(\left\|x_{1}\right\|^{2}+2\left\langle z, J x_{n}-J x_{1}\right\rangle\right)+\xi_{n} .
$$

This in turn implies from (3.10) that

$$
\lim _{n \rightarrow \infty} \phi\left(x_{n+1}, y_{n}\right)=0
$$

In view of (2.2), we see that

$$
\lim _{n \rightarrow \infty}\left(\left\|x_{n+1}\right\|-\left\|y_{n}\right\|\right)=0 .
$$

This in turn implies that

$$
\lim _{n \rightarrow \infty}\left\|y_{n}\right\|=\|\bar{x}\|
$$

It follows that

$$
\lim _{n \rightarrow \infty}\left\|J y_{n}\right\|=\|J \bar{x}\|
$$

This implies that $\left\{J y_{n}\right\}$ is bounded. Note that both $E$ and $E^{*}$ are reflexive. We may assume that $J y_{n} \rightarrow y^{*} \in E^{*}$. In view of the reflexivity of $E$, we see that $J(E)=E^{*}$. This shows that there exists an element $y \in E$ such that $J y=y^{*}$. It follows that

$$
\begin{aligned}
\phi\left(x_{n+1}, y_{n}\right) & =\left\|x_{n+1}\right\|^{2}-2\left\langle x_{n+1}, J y_{n}\right\rangle+\left\|y_{n}\right\|^{2} \\
& =\left\|x_{n+1}\right\|^{2}-2\left\langle x_{n+1}, J y_{n}\right\rangle+\left\|J y_{n}\right\|^{2} .
\end{aligned}
$$

Taking $\liminf _{n \rightarrow \infty}$ on the both sides of the equality above yields that

$$
\begin{aligned}
0 & \geq\|\bar{x}\|^{2}-2\left\langle\bar{x}, y^{*}\right\rangle+\left\|y^{*}\right\|^{2} \\
& =\|\bar{x}\|^{2}-2\langle\bar{x}, J y\rangle+\|J y\|^{2} \\
& =\|\bar{x}\|^{2}-2\langle\bar{x}, J y\rangle+\|y\|^{2} \\
& =\phi(\bar{x}, y) .
\end{aligned}
$$

That is, $\bar{x}=y$, which in turn implies that $y^{*}=J \bar{x}$. It follows that $J y_{n} \rightarrow J \bar{x} \in E^{*}$. Since $E^{*}$ enjoys the Kadec-Klee property, we obtain from (3.12) that

$$
\lim _{n \rightarrow \infty} J y_{n}=J \bar{x}
$$

Notice that

$$
\left\|J x_{n}-J y_{n}\right\| \leq\left\|J x_{n}-J \bar{x}\right\|+\left\|J \bar{x}-J y_{n}\right\| .
$$


It follows that

$$
\lim _{n \rightarrow \infty}\left\|J x_{n}-J y_{n}\right\|=0 \text {. }
$$

In view of

$$
\left\|J\left(T^{n} x_{n}\right)-J x_{n}\right\| \leq \frac{1}{1-\alpha_{n}}\left\|J y_{n}-J x_{n}\right\|+\frac{\alpha_{n}}{1-\alpha_{n}}\left\|J x_{n}-J x_{1}\right\|,
$$

we find from $\lim _{n \rightarrow \infty} \alpha_{n}=0$ that

$$
\lim _{n \rightarrow \infty}\left\|J\left(T^{n} x_{n}\right)-J x_{n}\right\|=0
$$

Notice that

$$
\left\|J\left(T^{n} x_{n}\right)-J \bar{x}\right\| \leq\left\|J\left(T^{n} x_{n}\right)-J x_{n}\right\|+\left\|J x_{n}-J \bar{x}\right\| .
$$

This implies from (3.14) that

$$
\lim _{n \rightarrow \infty}\left\|J\left(T^{n} x_{n}\right)-J \bar{x}\right\|=0 .
$$

The demicontinuity of $J^{-1}: E^{*} \rightarrow E$ implies that $T^{n} x_{n} \rightarrow \bar{x}$. Note that

$$
\left|\left\|T^{n} x_{n}\right\|-\|\bar{x}\|\right|=\left|\left\|J\left(T^{n} x_{n}\right)\right\|-\|J \bar{x}\|\right| \leq\left\|J\left(T^{n} x_{n}\right)-J \bar{x}\right\| .
$$

In view of (3.15), we see that $\lim _{n \rightarrow \infty}\left\|T^{n} x_{n}\right\|=\|\bar{x}\|$. Since $E$ has the Kadec-Klee property, we find that

$$
\lim _{n \rightarrow \infty}\left\|T^{n} x_{n}-\bar{x}\right\|=0
$$

Notice that

$$
\left\|T^{n+1} x_{n}-\bar{x}\right\| \leq\left\|T^{n+1} x_{n}-T^{n} x_{n}\right\|+\left\|T^{n} x_{n}-\bar{x}\right\| .
$$

In view of the asymptotic regularity of $T$, we find from (3.16) that

$$
\lim _{n \rightarrow \infty}\left\|T^{n+1} x_{n}-\bar{x}\right\|=0,
$$

that is, $T T^{n} x_{n}-\bar{x} \rightarrow 0$ as $n \rightarrow \infty$. It follows from the closedness of $T$ that $T \bar{x}=\bar{x}$.

Finally, we show that $\bar{x}=\Pi_{F(T)} x_{1}$. Letting $n \rightarrow \infty$ in (3.9), we arrive at

$$
\left\langle\bar{x}-w, J x_{1}-J \bar{x}\right\rangle \geq 0, \quad \forall w \in F(T) .
$$

It follows from Lemma 2.6 that $\bar{x}=\Pi_{F(T)} x_{1}$. This completes the proof of the theorem.

Remark 3.2 Since every uniformly smooth and uniformly convex Banach space is a reflexive, strictly convex, and smooth Banach space such that both $E$ and $E^{*}$ have the Kadec-Klee property, we find that Theorem 3.1 is still valid in the framework of uniformly smooth and uniformly convex Banach spaces. 
If $E$ is a Hilbert space, then we have the following result.

Corollary 3.3 Let E be a Hilbert space. Let $C$ be a nonempty closed and convex subset of E. Let $T: C \rightarrow C$ be a closed quasi-nonexpansive mapping in the intermediate sense. Assume that $T$ is asymptotically regular on $C$ and $F(T)$ is nonempty and bounded. Let $\left\{x_{n}\right\}$ be a sequence generated in the following manner:

$$
\left\{\begin{array}{l}
x_{0} \in E \quad \text { chosen arbitrarily, } \\
C_{1}=C \\
x_{1}=P_{C_{1} x_{0}} \\
y_{n}=\alpha_{n} x_{1}+\left(1-\alpha_{n}\right) T^{n} x_{n}, \\
C_{n+1}=\left\{z \in C_{n}:\left\|y_{n}-z\right\|^{2} \leq\left\|z-x_{n}\right\|^{2}+\alpha_{n}\left(\left\|x_{1}\right\|^{2}+2\left\langle z, x_{n}-x_{1}\right\rangle\right)+\xi_{n}\right\}, \\
x_{n+1}=P_{C_{n+1}} x_{1},
\end{array}\right.
$$

where

$$
\xi_{n}=\max \left\{0, \sup _{p \in F\left(T_{i}\right), x \in C}\left(\left\|p-T_{i}^{n} x\right\|^{2}-\|p-x\|^{2}\right)\right\} .
$$

If the sequence $\left\{\alpha_{n}\right\}$ satisfies the restriction $\lim _{n \rightarrow \infty} \alpha_{n}=0$, then the sequence $\left\{x_{n}\right\}$ converges strongly to $P_{F(T)} x_{1}$, where $P_{F(T)}$ is the metric projection from $C$ onto $F(T)$.

If $T$ is quasi- $\phi$-nonexpansive, then we have the following result.

Corollary 3.4 Let E be a reflexive, strictly convex, and smooth Banach space such that both $E$ and $E^{*}$ have the Kadec-Klee property. Let $C$ be a nonempty closed and convex subset of $E$. Let $T: C \rightarrow C$ be a closed quasi- $\phi$-nonexpansive mapping with a nonempty fixed point set. Let $\left\{x_{n}\right\}$ be a sequence generated in the following manner:

$$
\left\{\begin{array}{l}
x_{0} \in E \quad \text { chosen arbitrarily, } \\
C_{1}=C \\
x_{1}=\Pi_{C_{1} x_{0}} \\
y_{n}=J^{-1}\left(\alpha_{n} J x_{1}+\left(1-\alpha_{n}\right) J T x_{n}\right) \\
C_{n+1}=\left\{z \in C_{n}: \phi\left(z, y_{n}\right) \leq \phi\left(z, x_{n}\right)+\alpha_{n}\left(\left\|x_{1}\right\|^{2}+2\left\langle z, J x_{n}-J x_{1}\right\rangle\right)\right\} \\
x_{n+1}=\Pi_{C_{n+1}} x_{1} .
\end{array}\right.
$$

If the sequence $\left\{\alpha_{n}\right\}$ satisfies the restriction $\lim _{n \rightarrow \infty} \alpha_{n}=0$, then the sequence $\left\{x_{n}\right\}$ converges strongly to $\Pi_{F(T)} x_{1}$, where $\Pi_{F(T)}$ is the generalized projection from $C$ onto $F(T)$.

Next, we consider an equilibrium problem based on the projection algorithm. Find $p \in C$ such that

$$
f(p, y) \geq 0, \quad \forall y \in C
$$

We use $\operatorname{EP}(f)$ to denote the solution set of the equilibrium problem (3.17). That is,

$$
\mathrm{EP}(f)=\{p \in C: f(p, y) \geq 0, \forall y \in C\} .
$$


Given a mapping $Q: C \rightarrow E^{*}$, let

$$
f(x, y)=\langle Q x, y-x\rangle, \quad \forall x, y \in C
$$

Then $p \in \operatorname{EP}(f)$ if and only if $p$ is a solution of the following variational inequality. Find $p$ such that

$$
\langle Q p, y-p\rangle \geq 0, \quad \forall y \in C .
$$

For studying the equilibrium problem (3.17), let us assume that $f$ satisfies the following conditions:

(A1) $f(x, x)=0, \forall x \in C$;

(A2) $f$ is monotone, i.e., $f(x, y)+f(y, x) \leq 0, \forall x, y \in C$;

(A3)

$$
\limsup _{t \downarrow 0} f(t z+(1-t) x, y) \leq f(x, y), \quad \forall x, y, z \in C ;
$$

(A4) for each $x \in C, y \mapsto f(x, y)$ is convex and weakly lower semi-continuous.

Lemma 3.5 Let $C$ be a closed convex subset of a smooth, strictly convex, and reflexive Banach space E. Let $f$ be a bifunction from $C \times C$ to $\mathbb{R}$ satisfying (A1)-(A4). Let $r>0$ and $x \in E$. Then

(a) [37]. There exists $z \in C$ such that

$$
f(z, y)+\frac{1}{r}\langle y-z, J z-J x\rangle \geq 0, \quad \forall y \in C ;
$$

(b) [24,38]. Define a mapping $T_{r}: E \rightarrow C$ by

$$
S_{r} x=\left\{z \in C: f(z, y)+\frac{1}{r}\langle y-z, J z-J x\rangle, \forall y \in C\right\} .
$$

Then the following conclusions hold:

(1) $S_{r}$ is single-valued;

(2) $S_{r}$ is a firmly nonexpansive-type mapping, i.e., for all $x, y \in E$,

$$
\left\langle S_{r} x-S_{r} y, J S_{r} x-J S_{r} y\right\rangle \leq\left\langle S_{r} x-S_{r} y, J x-J y\right\rangle
$$

(3) $F\left(S_{r}\right)=\mathrm{EP}(f)$;

(4) $S_{r}$ is quasi- $\phi$-nonexpansive;

(5)

$$
\phi\left(q, S_{r} x\right)+\phi\left(S_{r} x, x\right) \leq \phi(q, x), \quad \forall q \in F\left(S_{r}\right)
$$

(6) $\mathrm{EP}(f)$ is closed and convex. 
Corollary 3.6 Let E be a reflexive, strictly convex, and smooth Banach space such that both $E$ and $E^{*}$ have the Kadec-Klee property. Let $C$ be a nonempty closed and convex subset of $E$. Let $f$ be a bifunction from $C \times C$ to $\mathbb{R}$ satisfying (A1)-(A4) with a nonempty solution set. Let $r$ be a positive real number. Let $\left\{x_{n}\right\}$ be a sequence generated in the following manner:

$$
\left\{\begin{array}{l}
x_{0} \in E \quad \text { chosen arbitrarily, } \\
C_{1}=C, \\
x_{1}=\Pi_{C_{1}} x_{0}, \\
y_{n}=J^{-1}\left(\alpha_{n} J x_{1}+\left(1-\alpha_{n}\right) J z_{n}\right), \\
C_{n+1}=\left\{z \in C_{n}: \phi\left(z, y_{n}\right) \leq \phi\left(z, x_{n}\right)+\alpha_{n}\left(\left\|x_{1}\right\|^{2}+2\left\langle z, J x_{n}-J x_{1}\right\rangle\right)\right\}, \\
x_{n+1}=\Pi_{C_{n+1}} x_{1},
\end{array}\right.
$$

where $z_{n}$ is such that

$$
f\left(z_{n}, s\right)+\frac{1}{r}\left\langle s-z_{n}, J z_{n}-J x_{n}\right\rangle \geq 0, \quad \forall s \in C .
$$

If the sequence $\left\{\alpha_{n}\right\}$ satisfies the restriction $\lim _{n \rightarrow \infty} \alpha_{n}=0$, then the sequence $\left\{x_{n}\right\}$ converges strongly to $\Pi_{\mathrm{EP}(f)} x_{1}$, where $\Pi_{\mathrm{EP}(f)}$ is the generalized projection from $C$ onto $\mathrm{EP}(f)$.

Proof Put $z_{n}=S_{r} x_{n}$. In view of Lemma 3.5, we find that $S_{r}$ is quasi- $\phi$-nonexpansive which is an asymptotically quasi- $\phi$-nonexpansive. We immediately find from Theorem 3.1 the desired conclusion.

\section{Competing interests}

The authors declare that they have no competing interests.

\section{Authors' contributions}

All authors contributed equally and significantly in writing this paper. All authors read and approved the final manuscript.

\section{Author details}

'School of Mathematics and Information Science, North China University of Water Resources and Electric Power, Zhengzhou, 450011, China. ${ }^{2}$ Department of Mathematics, Huanghuai University, Zhumadian, 463000, China.

\section{Acknowledgements}

The authors are grateful to the editor and the reviewers for their useful suggestions which improved the contents of the paper.

Received: 20 March 2013 Accepted: 3 May 2013 Published: 27 May 2013

\section{References}

1. Byrne, C: A unified treatment of some iterative algorithms in signal processing and image reconstruction. Inverse Probl. 20, 103-120 (2008)

2. Vanderluge, A: Optical Signal Processing. Wiley, New York (2005)

3. Qin, X, Chang, SS, Kang, SM: Iterative methods for generalized equilibrium problems and fixed point problems with applications. Nonlinear Anal. 11, 2963-2972 (2010)

4. Luo, H, Wang, Y: Iterative approximation for the common solutions of a infinite variational inequality system for inverse-strongly accretive mappings. J. Math. Comput. Sci. 2, 1660-1670 (2012)

5. Zegeye, $\mathrm{H}$, Shahzad, N: Strong convergence theorem for a common point of solution of variational inequality and fixed point problem. Adv. Fixed Point Theory 2, 374-397 (2012)

6. Cho, SY, Kang, SM: Approximation of fixed points of pseudocontraction semigroups based on a viscosity iterative process. Appl. Math. Lett. 24, 224-228 (2011)

7. Ye, J, Huang, J: Strong convergence theorems for fixed point problems and generalized equilibrium problems of three relatively quasi-nonexpansive mappings in Banach spaces. J. Math. Comput. Sci. 1, 1-18 (2011)

8. Cho, SY, Kang, SM, Qin, X: Hybrid projection algorithms for treating common fixed points of a family of demicontinuous pseudocontractions. Appl. Math. Lett. 25, 854-857 (2012) 
9. Noor, MA, Noor, Kl, Waseem, M: Decomposition method for solving system of linear equations. Eng. Math. Lett. 2, 34-41 (2013)

10. Cho, SY, Kang, SM, Kang, SM: Approximation of common solutions of variational inequalities via strict pseudocontractions. Acta Math. Sci. 32, 1607-1618 (2012)

11. Qin, X, Cho, SY, Kang, SM: Strong convergence of shrinking projection methods for quasi- $\boldsymbol{\phi}$-nonexpansive mappings and equilibrium problems. J. Comput. Appl. Math. 234, 750-760 (2010)

12. Genel, A, Lindenstrass, J: An example concerning fixed points. Isr. J. Math. 22, 81-86 (1975)

13. Haugazeau, Y: Sur les inéquations variationnelles et la minimisation de fonctionnelles convexes. Ph.D. thesis, Université de Paris (1968)

14. Goebel, K, Kirk, WA: A fixed point theorem for asymptotically nonexpansive mappings. Proc. Am. Math. Soc. 35, $171-174(1972)$

15. Bruck, RE, Kuczumow, T, Reich, S.: Convergence of iterates of asymptotically nonexpansive mappings in Banach spaces with the uniform Opial property. Colloq. Math. 65, 169-179 (1993)

16. Halpern, B: Fixed points of nonexpanding maps. Bull. Am. Math. Soc. 73, 957-961 (1967)

17. Martinez-Yanes, C, Xu, HK: Strong convergence of the CQ method for fixed point iteration processes. Nonlinear Anal. 64, 2400-2411 (2006)

18. Cioranescu, I: Geometry of Banach Spaces, Duality Mappings and Nonlinear Problems. Kluwer Academic, Dordrecht (1990)

19. Alber, Yal: Metric and generalized projection operators in Banach spaces: properties and applications. In: Kartsatos, AG (ed.) Theory and Applications of Nonlinear Operators of Accretive and Monotone Type. Dekker, New York (1996)

20. Reich, S: A weak convergence theorem for the alternating method with Bregman distance. In: Kartsatos, AG (ed.) Theory and Applications of Nonlinear Operators of Accretive and Monotone Type. Dekker, New York (1996)

21. Butnariu, D, Reich, S, Zaslavski, AJ: Asymptotic behavior of relatively nonexpansive operators in Banach spaces. J. Appl. Anal. 7, 151-174 (2001)

22. Agarwal, RP, Cho, YJ, Qin, X: Generalized projection algorithms for nonlinear operators. Numer. Funct. Anal. Optim. 28 1197-1215 (2007)

23. Qin, X, Su, Y, Wu, C, Liu, K: Strong convergence theorems for nonlinear operators in Banach spaces. Commun. Appl. Nonlinear Anal. 14, 35-50 (2007)

24. Qin, $X$, Cho, YJ, Kang, SM: Convergence theorems of common elements for equilibrium problems and fixed point problems in Banach spaces. J. Comput. Appl. Math. 225, 20-30 (2009)

25. Zhou, H, Gao, G, Tan, B: Convergence theorems of a modified hybrid algorithm for a family of quasi- $\phi$-asymptotically nonexpansive mappings. J. Appl. Math. Comput. 32, 453-464 (2010)

26. Qin, X, Cho, SY, Kang, SM: On hybrid projection methods for asymptotically quasi- $\phi$-nonexpansive mappings. Appl. Math. Comput. 215, 3874-3883 (2010)

27. Qin, X, Agarwal, RP: Shrinking projection methods for a pair of asymptotically quasi- $\phi$-nonexpansive mappings. Numer. Funct. Anal. Optim. 31, 1072-1089 (2010)

28. Qin, X, Su, Y: Strong convergence theorem for relatively nonexpansive mappings in a Banach space. Nonlinear Anal. 67, 1958-1965 (2007)

29. Qin, X, Wang, L: On asymptotically quasi- $\phi$-nonexpansive mappings in the intermediate sense. Abstr. Appl. Anal. 2012, Article ID 636217 (2012)

30. Cho, YJ, Qin, X, Kang, SM: Strong convergence of the modified Halpern-type iterative algorithms in Banach spaces. An. Univ. "Ovidius" Constanţa, Ser. Mat. 17, 51-68 (2009)

31. Chen, JW, Cho, YJ, Wan, Z: Shrinking projection algorithms for equilibrium problems with a bifunction defined on the dual space of a Banach space. Fixed Point Theory Appl. 2011, Article ID 91 (2011)

32. Qin, X, Cho, YJ, Kang, SM, Zhou, H: Convergence of a modified Halpern-type iteration algorithm for quasi- $\boldsymbol{\phi}$-nonexpansive mappings. Appl. Math. Lett. 22, 1051-1055 (2009)

33. Chang, SS, Lee, HWJ, Chan, CK, Yang, L: Approximation theorems for total quasi- $\phi$-asymptotically nonexpansive mappings with applications. Appl. Math. Comput. 218, 2921-2931 (2011)

34. Wei, L, Cho, YJ, Zhou, HY: A strong convergence theorem for common fixed points of two relatively nonexpansive mappings and its applications. J. Appl. Math. Comput. 29, 95-103 (2009)

35. Kim, JK: Strong convergence theorems by hybrid projection methods for equilibrium problems and fixed point problems of the asymptotically quasi- $\boldsymbol{\phi}$-nonexpansive mappings. Fixed Point Theory Appl. 2011 , Article ID 10 (2011)

36. Qin, X, Huang, S: On the convergence of hybrid projection algorithms for asymptotically quasi- $\boldsymbol{\phi}$-nonexpansive mappings. Comput. Math. Appl. 61, 851-859 (2011)

37. Blum, E, Oettli, W: From optimization and variational inequalities to equilibrium problems. Math. Stud. 63, 123-145 (1994)

38. Takahashi, W, Zembayashi, K: Strong and weak convergence theorems for equilibrium problems and relatively nonexpansive mappings in Banach spaces. Nonlinear Anal. 70, 45-57 (2009) 